\title{
Desarrollo de BenchBalance: un sistema para la evaluación de la capacidad de equilibrio en exoesqueletos robóticos
}

\author{
Cristina Bayón ${ }^{1,2}$, Gabriel Delgado-Oleas ${ }^{1}$, Nevio L. Tagliamonte ${ }^{3}$, Edwin van Asseldonk $^{2}$, Eduardo Rocon $^{1}$ \\ c.bayon@upm.es \\ ${ }^{1}$ Centro de Automática y Robótica, CSIC-UPM; ${ }^{2}$ University of Twente; ${ }^{3}$ Università Campus Bio-Medico di Roma
}

\section{Resumen}

Avances recientes en el control de exoesqueletos se están centrando en mejorar la asistencia de equilibrio y disminuir la dependencia de las muletas. Sin embargo, métodos apropiados para cuantificar la estabilidad de estos exoesqueletos (y de sus usuarios) aún son escasos. Una evaluación del equilibrio cuantitativa es fundamental para enriquecer el desempeño de los exoesqueletos y su interacción con los seres humanos. En este trabajo presentamos el sistema BenchBalance, una solución para realizar evaluaciones comparativas y reproducibles de equilibrio en exoesqueletos y sus usuarios. Al integrar dos elementos clave (perturbador manual y prenda inteligente), BenchBalance es una herramienta portátil y de bajo coste que proporciona una evaluación cuantitativa relacionada con la reacción y la capacidad de los exoesqueletos portátiles y sus usuarios para responder a perturbaciones externas controladas.

Palabras clave: Equilibrio; Evaluación; Exoesqueletos.

\section{INTRODUCCIÓN}

Los exoesqueletos robóticos de extremidades inferiores han ganado un gran interés en las últimas décadas, demostrando la capacidad de ayudar a personas con distintas discapacidades motoras a permanecer erguidas y poder desplazarse. Investigaciones recientes sobre estos dispositivos avanzan hacia el desarrollo de controladores que permitan mantener el equilibrio durante la bipedestación o la locomoción. Estos controladores, destinados a mejorar la estabilidad del usuario con el exoesqueleto, pueden variar en complejidad y van desde (1) extender el uso de estrategias de control desarrolladas en humanoides [2, 9, 11, 3], (2) utilizar enfoques bioinspirados $[10,13,1]$ o (3) implementar métodos heurísticos no complejos $[6,8,4]$. Sin embargo, hay una necesidad en la elaboración de procedimientos y métricas para cuantificar, comparar y evaluar adecuadamente la estabilidad de los diferentes controladores, exoesqueletos y sus usuarios.
El objetivo principal de esta contribución es mejorar los métodos actuales para evaluar las capacidades de equilibrio y estabilización de exoesqueletos de miembro inferior. Para ello, presentamos una nueva solución de benchmarking que permite realizar evaluaciones cuantitativas y reproducibles: BenchBalance (Figura 1). Nuestra solución es capaz de proporcionar y medir perturbaciones externas efectuadas manualmente por un experimentador, en términos de magnitud, orientación y ubicación, a sujetos que llevan un exoesqueleto. Las perturbaciones proporcionadas se pueden generar en cualquier grado de libertad de los tres planos de movimiento. Esta herramienta puede usarse para derivar métricas que cuantifiquen las capacidades de equilibrio del usuario que use (o no) un exoesqueleto. Para la fabricación y el desarrollo de BenchBalance se utilizaron componentes básicos existentes y materiales impresos en $3 \mathrm{D}$, por lo que su reproducción está asegurada para aquellos grupos de investigación interesados en la evaluación comparativa del equilibrio perturbado.

\section{SISTEMA BENCHBALANCE}

Para poder proporcionar una evaluación de equilibrio precisa y derivar métricas cuantitativas de las capacidades de equilibrio de un humano vistiendo un exoesqueleto, nos centramos en tres factores clave: primero, era necesario cuantificar la perturbación aplicada en términos de magnitud y orientación de la fuerza; en segundo lugar, era fundamental realizar una estimación correcta de dónde se aplica la perturbación, ya que las estrategias de equilibrio podrían diferir dependiendo de la ubicación de dicha perturbación; y tercero, era necesario evaluar la sincronización de la perturbación con la respuesta del usuario. En base a eso, el sistema BenchBalance (Figura 1) está compuesto por (1) un perturbador portátil de mano equipado con diferentes sensores, utilizado para proporcionar y cuantificar perturbaciones bien definidas en la parte superior del cuerpo humano; y (2) un detector de sistema de posición (prenda inteligente), utilizado para determinar la ubicación de la perturbación generada en relación con el ser humano que lleva el exoesqueleto. La información de 

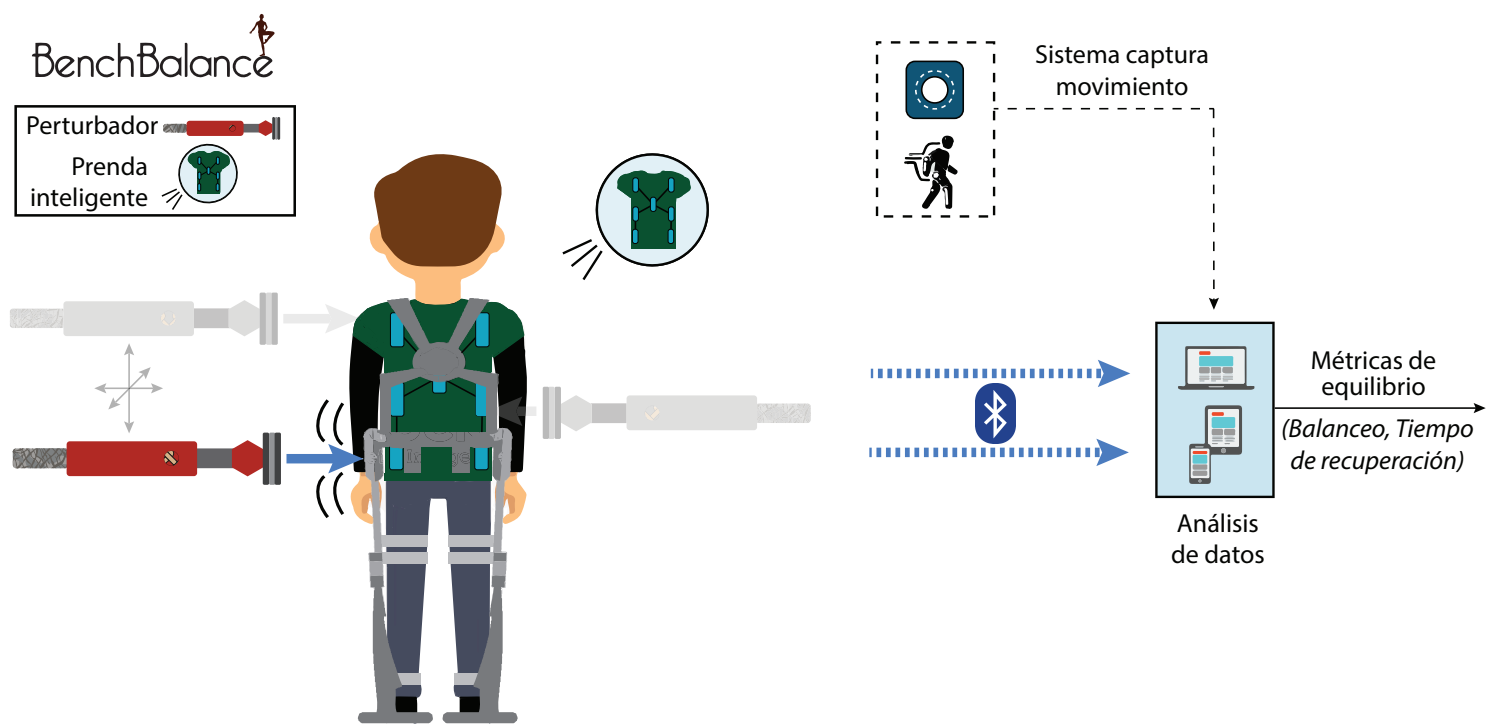

Figura 1: Esquema general del sistema BenchBalance. La información del perturbador y de la prenda inteligente se transmite por bluetooth, y se combina con la proporcionada por un sistema de captura de movimiento para su procesamiento

la fuerza externa proporcionada (magnitud, dirección y ubicación) se combina posteriormente con los datos cinemáticos del exoesqueleto o de un sistema de captura de movimiento (Mo-Cap) para generar las métricas de evaluación del equilibrio. En la evaluación, la reacción del usuario y exoesqueleto se relacionan con el tiempo empleado para recuperar la postura natural después de la perturbación y con la magnitud del balanceo corporal inducido.

\subsection{PERTURBADOR MANUAL}

El perturbador (Figura 2-(a)) fue diseñado para ser manipulado fácilmente por un operador, por lo que la versatilidad del dispositivo es muy alta en términos de usabilidad. Dicho sistema está equipado con los siguientes sensores:

- Sensor de fuerza (ME Systeme - MEK3D60a), que cuantifica la fuerza de tal perturbación aplicada al usuario en los 3 ejes

- Sensor inercial (Xsens-Mti-3 AHRS), para medir la orientación relativa de la perturbación con respecto al humano

\subsection{PRENDA INTELIGENTE}

Un chaleco textil fue diseñado y equipado con fibras ópticas poliméricas (Figura 2-(b)), con el fin de detectar el área de aplicación de la perturbación en el usuario. Esta prenda inteligente ofrece una solución robusta, mínimamente invasiva y multiplexada para controlar la presión de la perturbación sobre la parte superior del cuerpo humano. Está equipado con los siguientes sensores:
- Sensores de fibra óptica (PMMA-HFBREUS100Z, Broadcom Limited) distribuidos para cubrir el área frontal y posterior del tronco, el área debajo de los brazos y los hombros. En total, treinta sensores componen la prenda, cada uno con una lámpara flexible. $\mathrm{Su}$ funcionamiento está basado en la variación de la intensidad de la luz, convirtiendo esta intensidad en voltaje [7]. Así, se puede determinar la zona de presión de la perturbación

- Un sensor inercial similar al del perturbador (Xsens - MTi-3 AHRS), el cual se usa para calcular la orientación relativa del perturbador con respecto al cuerpo humano

\subsection{ANÁLISIS Y CAPTURA DE DATOS}

\subsubsection{Registro y sincronización}

Para facilitar la utilización del sistema BenchBalance, se desarrolló una interfaz de usuario. Esta interfaz recibe los datos del perturbador y la prenda inteligente a través de Bluetooth 5.0, y guía al experimentador a lo largo de los pasos necesarios para realizar perturbaciones reproducibles en el cuerpo humano. La interfaz está programada en Python (Python Software Foundation, Delaware, Estados Unidos), e incluye propiedades anónimas (altura, masa total) de los sujetos de prueba. Antes de iniciar las mediciones, se requiere calibrar el sistema. Esto también se hace a través de la interfaz, la cual indica al experimentador cómo colocar el perturbador y la prenda inteligente en la posición de calibración y durante cuánto tiempo, hasta 


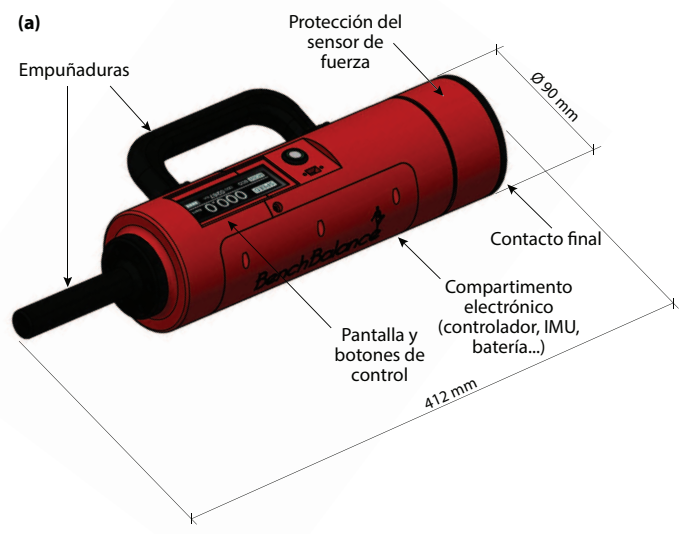

(b)

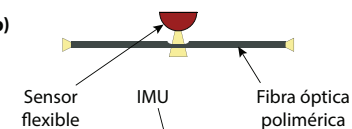

Zonas de actuació

Torso superior Espalda superior Torso medio Espalda media Torso izquierdo Hombro izquierdo flexible polimérica Torso derecho Hombro derecho

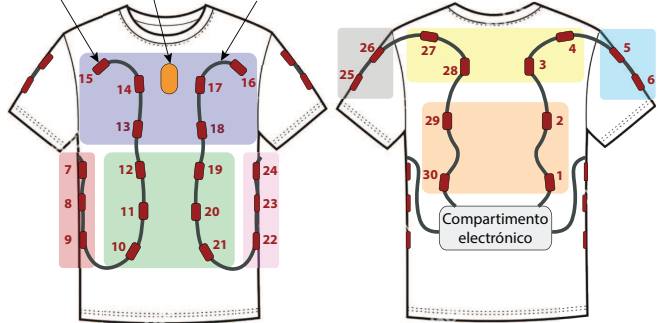

Figura 2: Subsistemas de BenchBalance: (a) perturbador manual y (b) prenda inteligente

que se hayan eliminado los valores de compensación. Después de calibrar el sistema, el experimentador tiene la opción de registrar libremente o de seguir un protocolo predefinido de perturbaciones.

Los datos registrados con la interfaz se envían a una computadora host para su posprocesamiento, sincronizándose correctamente mediante "timestamp".

\subsubsection{Métricas}

El objetivo de las métricas generadas es la cuantificación de la respuesta a la perturbación aplicada en condiciones de bipedestación, expresando el análisis en función de variables como la magnitud del balanceo corporal y el tiempo de recuperación del usuario+exoesqueleto. Para poder generar estás métricas, es necesario obtener variables controladas del sistema en las que se incluyen:

- Magnitud de la perturbación: amplitud máxima de la fuerza aplicada al sujeto expresada en Newtons (magnitud del vector resultante de los tres ejes)

- Tipo de Perturbación: nivel de perturbación que depende del peso del sujeto. Se definieron dos niveles: "Débil" ( $8 \pm 2 \%$ peso del usuario+exoesqueleto) y "Fuerte" $(16 \pm 2 \%$ peso del usuario+exoesqueleto)

- Duración de la perturbación: tiempo de aplicación de la fuerza. Se definió un nivel aceptable entre 0.2 y 0.5 segundos

- Orientación: ángulo relativo entre el cuerpo del sujeto y el perturbador. Se requiere una orientación lo más perpendicular posible al tronco humano, con una tolerancia de \pm 15 grados

- Ubicación: zona de la prenda inteligente donde se provoca la perturbación al sujeto

- Exoesqueleto: valor booleano representando si el usuario viste o no el exoesqueleto

Con dichas variables controladas se calculan posteriormente los indicadores o métricas de equilibrio:

- Balanceo del Cuerpo: representa el ángulo máximo del cuerpo en respuesta a una perturbación proporcionada. Un valor alto del balanceo del cuerpo indica una menor capacidad del sujeto para mantener el equilibrio

- Tiempo de recuperación: representa el tiempo empleado para recuperarse de una perturbación (el centro de masa, CoM, vuelve a la posición de reposo). Se considera que la posición se ha recuperado si la velocidad de oscilación del CoM es menor que un umbral de $0.015 \mathrm{rad} / \mathrm{s}$ después del inicio de la perturbación

Para calcular las métricas de equilibrio, en BenchBalance consideramos un modelo del ser humano (y el exoesqueleto) compuesto por un sistema de cuerpo rígido de cinco segmentos (Figura 3). Las articulaciones entre segmentos se consideran como articulaciones rotativas puras. Se supone que los pies no pueden despegarse del suelo y, por lo tanto, no están incluidos en el modelo (i.e. la articulación del tobillo se supone virtualmente conectada directamente con el suelo). 

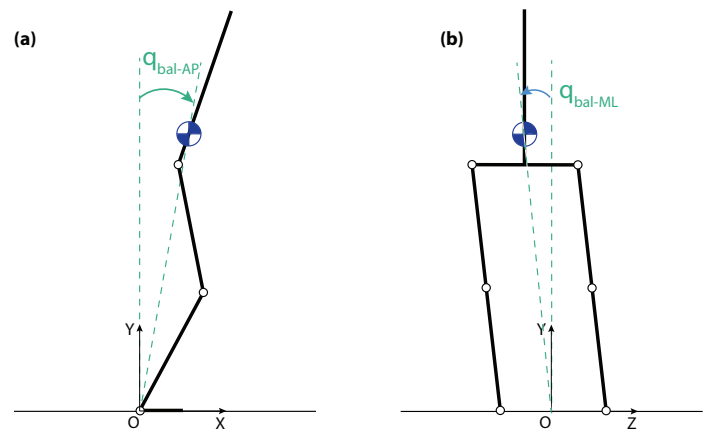

(c)
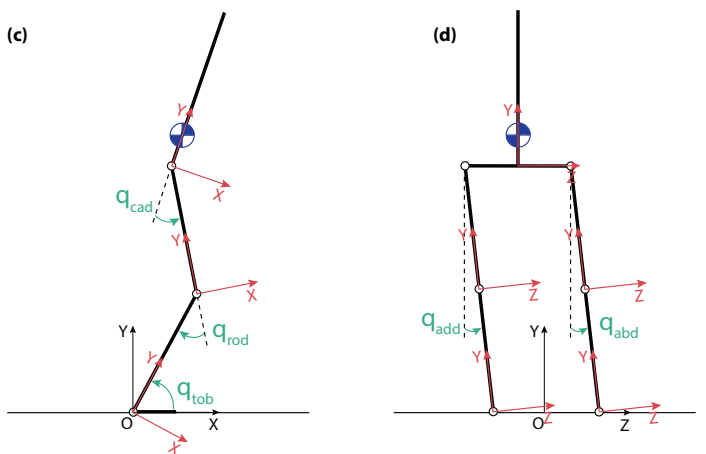

Figura 3: Convención de ángulos articulares $\left(q_{c a d}, q_{\text {rod }}, q_{t o b}, q_{a d d / a b d}\right)$ y ángulo de balanceo $\left(q_{b a l}\right)$ en las direcciones antero-posterior (AP) y medio-lateral (ML)

Como parámetros de entrada para los algorithmos de BenchBalance, se requiere: (1) la longitud de los segmentos considerados (pantorrilla, muslo y tronco [5]), lo cual se puede medir directamente en el cuerpo del sujeto; (2) las masas de cada segmento, que se calculan a partir de la masa total del sujeto utilizando la aproximación de Winter [12]. Si el exoesqueleto está presente, las masas y propiedades del dispositivo robótico se deberán proporcionar a través de un archivo en formato "Unified Robot Description Format" (urdf); y (3) el CoM de cada segmento, calculado en base a [12] con respecto a la articulación distal.

En el modelo, se toman algunas simplificaciones para los planos sagital y frontal (Figura 3):

- Para el plano sagital, las piernas izquierda y derecha se consideran idénticas y colocadas simétricamente. Además, solo se consideran los ángulos puros de flexión y extensión de cadera, rodilla y tobillo $\left(q_{c a d}, q_{\text {rod }}, q_{t o b}\right)$

- Para el plano frontal, la adducción-abducción de la cadera $\left(q_{a d d / a b d}\right)$ se considera igual para las articulaciones de cadera izquierda y derecha

El CoM total (exoesqueleto + humano) de un segmento $i\left({ }^{i} C o M_{H E}\right)$, se calcula del siguiente modo:

$$
{ }^{i} C_{H E} M_{H E}=\frac{{ }^{i} \operatorname{CoM}_{H}{ }^{i} M_{H}+{ }^{i} \operatorname{CoM}_{E}{ }^{i} M_{E}}{{ }^{i} M_{H}+{ }^{i} M_{E}}
$$

donde ${ }^{i} C_{o} M_{H}$ y ${ }^{i} C o M_{E},{ }^{i} M_{H}$ y ${ }^{i} M_{E}$ corresponden al CoM y masa del segmento $i$ del ser humano (H) y el exoesqueleto (E), respectivamente.

\section{CONCLUSIONES}

BenchBalance aporta a la comunidad científica la posibilidad de obtener métricas estándar para evaluar la estabilidad y el control de equilibrio con exoesqueletos robóticos, siendo también posible su extensión a robots humanoides. Además, con BenchBalance, se abren diversas posibilidades para evaluar sistemas en los que sean necesarias perturbaciones conocidas, tanto en magnitud como en orientación. A su vez, la prenda inteligente puede ser utilizada en diferentes escenarios en los que sea necesario conocer zonas de presión en el cuerpo del usuario.

Las métricas de equilibrio derivadas (ángulo de balanceo y tiempo de recuperación) aportan un novedoso enfoque para evaluar de forma objetiva la estabilidad de una persona con o sin exoesqueleto. Esto, a su vez, puede aportar grandes beneficios para la comparación de controladores de equilibrio en exoesqueletos robóticos.

El sistema BenchBalance ha sido desarrollado para el consorcio EUROBENCH y se incorporará como un banco de pruebas en sus laboratorios para futuros experimentos de comparación de exoesqueletos portátiles.

\section{Agradecimientos}

Esta investigación ha sido financiada por el proyecto europeo EUROBENCH2020 (eurobench2020.eu), número de subvención n779963.

\section{English summary}

\section{BenchBalance: a system to assess benchmarking balance capabilities of wearable robots}

\begin{abstract}
Recent advances on the control of exoskeletons are being centered on improving balance support and decreasing the reliance on crutches. However, appropriate methods to quantify the stability of these exoskeletons (and and their users) are still under development. A reliable and reproducible balan-
\end{abstract}


ce assessment is critical to enrich exoskeletons' performances and their interaction with human beings. In this work we present the BenchBalance system, a benchmarking solution to conduct reproducible balance assessments of exoskeleton and users. Integrating two key elements (manual perturbator and smart garment), BenchBalance is a portable and low-cost tool that provides a quantitative assessment related to the reaction and capacity of wearable exoskeletons and their users to respond to controlled external perturbations.

Keywords: Balance; Assessment; Exoskeletons; Benchmarking.

\section{Referencias}

[1] Maarten Afschrift y col. "Mechanical effort predicts the selection of ankle over hip strategies in nonstepping postural responses". En: Journal of Neurophysiology 116.4 (2016), págs. 1937-1945. ISSN: 15221598. DOI: $10.1152 / j n .00127 .2016$.

[2] Ayush Agrawal y col. "First Steps Towards Translating HZD Control of Bipedal Robots to Decentralized Control of Exoskeletons". En: IEEE Access 5 (2017), págs. 9919-9934. ISSN: 21693536. DOI: 10 . 1109 / ACCESS . 2017.2690407.

[3] C. Bayón y col. "Can Momentum Based Control Predict Human Balance Recovery Strategies?" En: IEEE Transactions on Neural Systems \& Rehabilitation Engineering 28.9 (2020), págs. 2015-2024. DOI: 10. 1109/TNSRE. 2020.3005455.

[4] C. Bayón y col. "Cooperative ankleexoskeleton control can reduce effort to recover balance after unexpected disturbances during walking". En: submitted to Journal of NeuroEngineering and Rehabilitation (2021).

[5] EUROBENCH. Eurobench framework documentation. 2021. URL: https : / / eurobench . github . io / sofware _ documentation / latest / data _ format . html \# EurobenchDataFormat (visitado 10-05-2021).

[6] Rachel W Jackson y Steven H Collins. "Heuristic-Based Ankle Exoskeleton Control for Co-Adaptive Assistance of Human Locomotion". En: IEEE Transactions on
Neural Systems and Rehabilitation Engineering PP.c (2019), pág. 1. DOI: $10.1109 /$ TNSRE . 2019.2936383.

[7] Arnaldo G. Leal-Junior y col. "Multiplexing technique for quasi-distributed sensors arrays in polymer optical fiber intensity variation-based sensors". En: Optics and Laser Technology 111 (2019), págs. 81-88. ISSN: 00303992. DOI: 10 . $1016 / \mathrm{j}$. optlastec . 2018.09 .044$.

[8] V. Monaco y col. "An ecologically-controlled exoskeleton can improve balance recovery after slippage". En: Scientific Reports 7.May (2017), págs. 1-10. ISSN: 20452322. DOI: 10 . 1038/srep46721.

[9] Carlotta Mummolo y col. "Stability of Mina $\mathrm{V} 2$ for robot-assisted balance and locomotion". En: Frontiers in Neurorobotics 12.October (2018), págs. 1-16. ISSN: 16625218. DOI: $10.3389 /$ fnbot .2018 .00062 .

[10] Nitish Thatte y Hartmut Geyer. "Toward Balance Recovery with Leg Prostheses using Neuromuscular Model Control". En: IEEE Transactions on Biomedical Engineering (2015), págs. 1-10.

[11] Barkan Ugurlu y col. "Variable ankle stiffness improves balance control: experiments on a bipedal exoskeleton". En: IEEE Transactions on mechatronics 21.1 (2016), págs. 79-87.

[12] D.A. Winter. "Anthropometry". En: Biomechanics and Motor Control of Human Movements. 4. ${ }^{a}$ ed. John Wiley \& Sons Inc., 2009, págs. 82-106. ISBN: 9780470398180.

[13] Guoping Zhao y col. "Bio-Inspired Balance Control Assistance Can Reduce Metabolic Energy Consumption in Human Walking". En: IEEE Transactions on Neural Systems and Rehabilitation Engineering 27.9 (2019), págs. 1760-1769. DOI: 10 . 1109 / TNSRE . 2019.2929544.

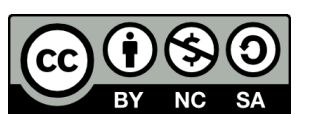

(C) 2021 by the authors. Submitted for possible open access publication under the terms and conditions of the Creative Commons Attribution CC BY-NC-SA 4.0 license (https://creativecommons.org/licenses/by-ncsa/4.0/deed.es). 